\title{
FGFR2 Rearrangement Analysis
}

National Cancer Institute

\section{Source}

National Cancer Institute. FGFR2 Rearrangement Analysis. NCI Thesaurus. Code C157192.

A procedure used to detect and identify rearrang ements involving the FGFR2 gene. 